Pacific Journal of Mathematics

REGULAR FF RINGS 


\title{
REGULAR FPF RINGS
}

\author{
S. PAGE
}

It is shown that a von Neumann regular ring is FPF (i.e., very faithful finitely generated module is a generator) iff it is self-injective of bounded index.

1. Introduction. An associative ring $R$ is called a left (F)PF ring if every (finitely generated) faithful module generates the category of left $R$-modules. Azumaya [1], Osofsky [7], and Utumi $[9,12]$ characterized the left $\mathrm{PF}$ rings as those rings for which any one of the following equivalent conditions holds:

$\left(\mathrm{PF}_{1}\right) \quad R$ is left self-injective, semiperfect, and has essential left socle.

$\left(\mathrm{PF}_{2}\right) \quad R$ is left self-injective with finitely generated essential left socle.

$\left(\mathrm{PF}_{3}\right) \quad R=\bigoplus \sum_{i=1}^{n} R e_{i}, e_{i}^{2}=e_{i}$ and $R e_{i}$ is injective with simple essential socle.

$\left(\mathrm{PF}_{4}\right) R$ is an injective cogenerator in $R$-mod.

$\left(\mathrm{PF}_{5}\right) R$ is left self-injective and every simple left $R$-module embeds in $R$.

C. Faith in [3, 4] has studied semiperfect left FPF rings. In this note we are concerned with von Neumann regular rings which are left FPF. As the conditions $\mathrm{PF}_{1}-\mathrm{PF}_{5}$ readily point out a von Neumann regular ring which is $\mathrm{PF}$ must be semi-simple artinian. In this note we show that if $R$ is von Neumann regular, then $R$ is FPF iff $R$ is of bounded index and left self-injective. It follows that for regular rings left FPF implies right FPF also.

II. Preliminaries. In what follows $R$ will denote an associative ring with unity and all modules will be unitary left $R$-modules unless otherwise noted.

A ring $R$ is von Neumann regular if for every $a \in R$ there is an $x \in R$ such that $a x a=a$. We will just say $R$ is regular.

Definition. For a set $S \subset M, M$ an $R$-module, let ${ }^{-} S=\{r \in R$ : $r s=0$ for all $s \in S\}$. If $M$ is a right $R$-module, define $S^{\perp}=\{r \in R$ : $s r=0$ for all $\mathrm{s} \in S\}$.

Definition. Let $M$ be an $R$-module. Let $Z(M)$ be the left singular submodule of $M$ i.e., $Z(M)$ is the set of elements of $M$ whose annihilators are essential left ideals of $R$. $M$ is called nonsingular if $Z(M)=0$. 
Definition. A ring $R$ is of bounded index if there exists an integer $N>0$ such that if $x^{n}=0$ then $x^{N}=0$.

Definition. Let $M$ and $N$ be $R$-modules. Let $N-\operatorname{dim} M=$ $\sup \left\{n: \oplus \sum_{i=1}^{n} N_{i} \subset M, N_{i} \cong N, i=1, \cdots, n\right\}$. Also, let $D(M)=$ $\sup \{N-\operatorname{dim} M, N \in R$-mod $\}$.

The following result of Utumi [10] gives the connection between rings of bounded index and FPF rings. We include the proof for completeness.

THEOREM 1. Let $R$ be a ring with zero singular left ideal. Then $R$ is of bounded index if $D(R)<\infty$ and in case $R$ is regular $D(R)$ equals the smallest bound on the index of nilpotence.

Proof. We can suppose $R$ is regular for the maximal ring of quotients, $Q(R)$, is regular and $R$ is an essential submodule of $Q(R)$. Suppose $x^{n}=0$ but $x^{n-1} \neq 0$, for some $x \in R$. Let $K_{1}={ }^{\perp}\left(x^{n-1}\right)$ and consider $0 \rightarrow K_{1} \rightarrow R \stackrel{x^{n-1}}{\longrightarrow} R x^{n-1} \rightarrow 0$. The sequence splits by regularity of $R$, so $R \supseteqq W_{1} \cong R x^{n-1}$ and $W_{1} \cap K_{1}=0$. Let $K_{2}={ }^{\perp}\left\{x^{n-2}\right\} \cap$ $R x$ and form $0 \rightarrow K_{2} \rightarrow R x \rightarrow R x^{n-1} \rightarrow 0$ which also splits. Therefore there exists $W_{2} \subseteq R x$ with $W_{2} \cap K_{2}=0$ and $w_{2} \cong R x^{n-1}$ so that $w_{2} \cong$ $W_{1}$. Also since $K_{1} \cap W_{1}=0$ and $R x \subset K_{1} W_{2} \cap W_{1}=0$.

By $n-1$ applications of the above technique we obtain $W_{1} \cong$ $W_{2} \cong \cdots \cong W_{n-1}$ with $R x^{n-1} \cong K_{i}={ }^{\perp}\left\{x^{n-i}\right\} \cap R x^{i}$, and $W_{i} \cap K_{i}=0$. It follows that $D(R) \geqq n$ since $\left(\oplus \sum_{i=1}^{n-1} W_{i}\right) \oplus R x^{n-1} \subset R$.

Next suppose $\left\{L_{i}\right\}_{i=1}^{n}$ is an independent set of left ideals in $R$ with $L_{i} \cong L_{j}$ for all $i$ and $j \leqq n$. Since $R$ is regular we can assume the $L_{i}$ are all idempotent generated, by $e_{1}, e_{2}, \cdots, e_{n}$, say, with $e_{i} e_{j}=0$ for $i, j=1, \cdots, n, i \neq j$. Let $\phi_{i j}: R e_{i} \cong R e_{j}$. Then $\phi_{i j}$ is right multiplication by $e_{i} r_{i j} e_{j}$ for some $r_{i j} \in R$. Let $x=\Sigma e_{i} r_{i, i+1} e_{i+1}$. Then $x^{n}=0$ but $x^{n-1} \neq 0$.

COROLlaRY 1.1. If $R$ is a domain which is not a left Ore domain, $Q(R)$ is of unbounded index, where $Q(R)$ is the maximal left quotient ring of $R$.

Another fundamental result is the following of Bumby [2].

Proposition 1.2. Let $M_{1}$ and $M_{2}$ be injective modules with $0 \rightarrow$ $M_{1} \rightarrow M_{2}$ and $0 \rightarrow M_{2} \rightarrow M_{1}$. Then $M_{1} \cong M_{2}$.

III. Regular FPF rings. We start with commutative rings, then using Morita equivalence build up to the more general case. 
THEOREM 2. The following are equivalent for a commutative regular ring $R$.

(i) $R$ is self-injective.

(ii) $R$ is FPF.

(iii) The trace of every finitely generated faithful module is finitely generated.

Proof. If $R$ is injective and $M$ is a finitely generated faithful module, then $R$ embeds in a finite direct sum of copies of $M$ as a direct summand. This gives (i) $\Rightarrow$ (ii).

That (ii) implies (iii) is trivial.

Assume (iii) and let $q \in Q$, the injective hull of $R$. Form $R q+$ $R=M$. Now trace $(M)$ is finitely generated since $M$ is finitely generated and faithful. Since $R$ is regular and trace $(M)$ is finitely generated, we have that trace $(M)=R e, e^{2}=e$. Let $i \in I=\{r \in R$ : $r q \in R\}$, an essential ideal. Then multiplication by $i$ defines a map of $M$ into $R$ and this map sends 1 into $i$ so $I \subset$ trace $(M)$. Now take $f: M \rightarrow R$. Let $f(q)=x_{0}$ and $f(1)=y_{0}$. Then for every $z \in I$ we have $f(z q)=z q y_{0}$ so $z\left(x_{0}-q y_{0}\right)=0$, hence $x_{0}=q y_{0}$ and $y_{0} \in I$. I is generated by idempotents so we can take $y_{0}=y_{0}^{2}$ so that $x_{0}=x_{0} y_{0}$, that is, trace $(M) \subseteq I$ too. Since $I=R e$ and $I$ is essential, $I=R$ and hence $q \in R$.

COROLLARY 2.1. If $R$ is a strongly regular ring (all idempotents are central) then $R$ is FPF iff $R$ is self-injective.

Proof. If $R$ is strongly regular left ideals are ideals and are generated by idempotents. Also if $M$ is finitely generated by $x_{1}$, $\cdots, x_{n}$ say $M=\bigcap_{i=1}^{n}\left\llcorner\left\{x_{i}\right\}\right.$ for strongly regular rings. With these observations the previous proof goes through.

If $D$ is a division ring and $R=\operatorname{End}_{D}(\gamma)$ then $R$ is FPF iff $\gamma$ is finite dimensional over $D$, but $R$ is always self-injective and regular. The significant observation is that if $\gamma$ is infinite dimensional over $D$ and $f \in R$ is a map with one dimensional range $R f$ is finitely generated and faithful but can not generate $R$ because roughly $R$ contains infinitely many copies of $R f$ i.e., $R f-\operatorname{dim} R=\infty$.

We do have the following.

Proposition 3. Let $R$ be a ring with $Z(R)=0$. If $R$ is left FPF then every left ideal is an essential submodule of a direct summand of $R$.

Proof. Let $L$ be any left ideal and $B$ a left ideal maximal with respect to $L \cap B=0$. Form $R / L \oplus R / B=M$. $M$ is faithful 
and finitely generated so generates $R$. Now if $f: M \rightarrow R$, let $f((1+$ $L, 0))=x_{0}$ and $f((0,1+B))=y_{0}$. Then $x_{0} \in L^{\perp}$ and $y_{0} \in B^{\perp}$ so since $M$ is faithful $L^{\perp}+B^{\perp}=R$. This gives ${ }^{\perp}\left(L^{\perp}+B^{\perp}\right)=0$ or ${ }^{\perp}\left(L^{\perp}\right) \cap$ ${ }^{\perp}\left(B^{\perp}\right)=0$. Since $L \subseteq{ }^{\perp}\left(L^{\perp}\right)$ and $B \subseteq{ }^{\perp}\left(B^{\perp}\right)$ the maximality of $B$ gives $B={ }^{\perp}\left(B^{\perp}\right)$. Also, if we now take $L_{1} \supset L$ and maximal with respect to $L_{1} \cap B=0, L_{1}$ is an essential extension of $L$, and ${ }^{\perp}\left(L_{1}^{\prime}\right)=$ $L_{1}$ as we have just seen. Now we have $0=\left(L_{1}+B\right)^{\perp}$ since $L_{1}+B$ is essential, hence $L_{1}^{\perp} \cap B^{\perp}=0$. Also $L_{1}^{\perp}+B^{\perp}=R$ by the above which yields $L_{1}^{\perp}=e R, e^{2}=e$ so that ${ }^{\perp}\left(L_{1}^{\perp}\right)=R(1-e)$ a direct summand, as promised.

Proposition 4. If $R$ is a regular ring which is left FPF, then $R$ is left self-injective.

Proof. If $R$ is regular then certainly $Z(R)=0$ and by Proposition 3 each left ideal is essential in a direct summand of $R$. In regular rings it is trivial that a left ideal isomorphic to a direct summand is a direct summand. These two properties constitute the definition of left continuous and the last corollary of Utumi [11, Corollary 8.4] states that if $R$ and any matrix ring over $R$ are both continuous $R$ is self-injecture. Since both FPF and regularity are easily checked to be Morita invariant properties, it follows that $R$ is left self-injective.

REMARK. The integers are FPF but lack the second part of the definition of left continuous.

Proposition 5. Let $\left\{R_{i}\right\}_{i \in I}$ be a collection of rings. Let $R=$ $\Pi_{i \in I} R_{i}$ as rings. Then $R$ is left FPF iff each $R_{i}$ is left FPF and for each collection $\left\{M_{i}: M_{i}\right.$ a finitely generated faithful $R_{i}$-module $i \in I\}$ such that $\pi M_{i}$ is a finitely generated $R$-module, there exists an integer $N>0$ such that $R_{i}$ is a homomorphic image of a direct sum of $N$ copies of $M_{i}$ for each $i \in I$.

Proof. Routine coordinate wise computation yields the proposition.

The previous proposition points out that if $R$ is a product of matrix rings over division rings in order that $R$ be left FPF the matrix rings had better not become to "large". It also suggests we look at the types given by Kaplansky and refined by Goodearl and Boyle [5].

Definition. A regular left self-injective ring $R$ is called type 
$I$ if for every direct summand $L$ of $R, L \supseteqq L^{1} \neq 0$, a left ideal, such that for any left ideals $A \neq 0$ and $B \neq 0$ contained in $L^{1}$, $\operatorname{Hom}(A, B) \neq 0$. If $L=L^{1} L$ is called abelian.

Definition. A ring $R$ is called Dedekind finite if $x y=1$ iff $y x=1$, otherwise we say $R$ is Dedekind infinite.

Definition. A regular left self-injective ring $R$ is called type $I I$ if $R$ contains an idempotent $e$ such that $R e$ is faithful, $e R e$ is Dedekind finite but $R$ contains no abelian left ideals.

Definition. A regular left self-injective ring $R$ is type $I I I$ if $0 \neq e^{2}=e$ then $e R e$ is not Dedekind finite.

Type III rings are characterized by the fact that for any direct summand, $L$, then $L \cong L \oplus L$.

Theorem [Kaplansky [6], Goodearl, Boyle [5, Corollary 7.7, p. 48]. If $R$ is a regular left self-injective ring, then $R=\prod_{i=1}^{5} R_{i}$, where $R_{1}$ is type $I$ and Dedekind finite, $R_{2}$ is type $I$ and Dedekind infinite, $R_{3}$ is type $I I$ and Dedekind finite, $R_{4}$ is type II and Dedekind infinite, and $R_{5}$ is type III.

Remark. All type $I I I$ rings are Dedekind infinite. Also, we will adopt Kaplansky [6, p. 11] notation and say $R$ is type $I_{f}$ if $R$ is type $I$ and Dedekind finite, type $I_{\infty}$ if type $I$ and Dedekind infinite, type $I I_{f}$ if $\cdots$, type $I I_{\infty} \cdots$.

Proposition 6. If $R$ is regular and FPF then $R$ is biregular.

Proof. Let $x \in R$. We wish to show $R x R$ is generated by a central idempotent. Let $H={ }^{\perp}(R x R)$. If $H=0$, then $R x$ generates so $R x R=R$. If $H \neq 0$, then $H$ is the left ideal maximal with respect to $H \cap R x R=0$. It follows that $H$ is a direct summand of $R$ because $R$ is self-injective. Now $H \oplus R x$ is a finitely generated faithful module, hence a generator, so trace $(H \oplus R x)=$ $H \oplus R x R=R$.

Proposition 7. If $R$ is regular left FPF, $R$ is Dedekind finite.

Proof. If not, then by [5, Prop. 7.4, p. 48] $R=R_{1} \times R_{2}$ with $R_{2} \neq 0$ and purely infinite, i.e., for every $0 \neq e$, a central idempotent in $R_{2}, e R_{2} e$ is not Dedekind finite. So assume $R \neq 0$ and purely infinite. 
By [5, Thm. 6.2, p. 41] there is in $R$ a sequence of idempotents $e_{1}, e_{2}, \cdots$ such that for each $i, R e_{i} \cong R$, and $\sum_{i=1}^{\infty} R e_{i}$ is direct, essential and $R=E\left(\sum_{i=1}^{\infty} R e_{i}\right)$. Let $M=R / \sum_{i=1}^{\infty} R e_{i}$. We claim $M$ is faithful. If not, there exist $x \in R$ such that $R \times R \cong M$. By Proposition 6, $R x R=R e$ for some central idempotent $e$. Since $e M=0$ it follows that $R e \subseteq \sum_{i=1}^{\infty} R x_{i}$. But then $R e \subseteq \sum_{i=1}^{N} R x_{i}$ for some $N$ large enough. This implies $R \mathrm{e} \cap R x_{j}=0$ for $j>N$, which implies $e x_{j}=0 j>N$ since $e$ is central. However, since $R x_{i} \cong R x_{j}$ for all $i$ and $j$ and $e$ is central, then $e x_{i}=0$ for all $i$, a contradiction.

Thus $M$ is faithful. $M$ is also singular, hence $R$ is singular so must be zero.

COROLLARY 7.1. If $R$ is regular FPF type $I$, then $R$ is of bounded index.

Proof. By [5, p. 30] we see that if $R$ is type $I, R$ contains an idempotent such that $e R e$ is strongly regular and $R e$ is faithful. It follows that $R$ is Morita equivalent to a strongly regular ring. Then using Tominaga [8, Lemma 1, p. 139] we see that $R$ is of bounded index.

Proposition 8. Let $R$ be a regular left FPF ring of type $I I_{f}$. Then $R=\{0\}$.

Proof. Let $0 \neq R$ be as above. We claim $R$ can not be a simple ring. If $R$ were a simple ring since it is type $I I$ it cannot be a semi-simple ring, hence must have an essential left $E$. But then $R / E$ is faithful by the simplicity of $R$ hence a generator of $R$. This says $Z(R)=R$, ridiculous. Since $R$ is not simple there must exist an idempotent $e_{1} \in R$ such that $0 \neq R e_{1} R \neq R$. Now let $H_{1}={ }^{\perp}\left(R e_{1} R\right)$. If $H_{1}=0$ then $R e_{1}$ generates $R$ which it does not, so $H_{1} \neq 0 . \quad H_{1}$ is the left ideal maximal with respect to $H_{1} \cap R e_{1} R=0$, so $H_{1}$ is a summand by injectivity of $R$. It follows that $H_{1} \oplus$ $R e_{1} R=R$ as above. Now $H_{1}$ and $R e_{1} R$ are type $I I_{f}$ left FPF rings so we can repeat the process to $R e_{1} R$ to obtain an ideal $H_{2} \subseteq R e_{1} R$. Continuing in this way we obtain $H_{1} \oplus H_{2} \oplus \cdots \leqq R$ each $H_{i}$ a nonzero two sided direct summand of $R$. Since each $H_{i}$ is type $I I_{f}$ we can choose an idempotent $f_{i} \in H_{i}$ such that $H_{i}=\bigoplus \sum_{j=1}^{i} R f_{i_{j}}$, $R f_{i} \cong R f_{i_{j}}$ for all $j \leqq i$. Next take $R g=E\left(\oplus \Sigma H_{i}\right) . \quad R g$ is a two sided ideal for the hull of any two sided ideal in a semiprime left self-injective ring is complemented by its left annihilator which is a two sided ideal. We can assume then that $g$ is a central idempotent. Form $\prod_{i=1}^{\infty} R f_{i}$ and let $M$ be the cyclic submodule generated 
by $R\left(\left(f_{i}\right)_{i \in I}\right)$. Let $N=M \oplus R(1-g)$. Then $y N=0$ iff $y(g-1)=0$ and $y R f_{i}=0$ for all $i$, so $y R f_{i} R=0$ for all $i$. Then $y\left(\sum_{i=1}^{\infty} H_{i}\right)=0$. But since $y g=y$ there exists an essential left ideal $E$ such that $E y \subseteq \sum_{i=1}^{\infty} H_{i}$ and $(E y)^{2}=0$ implies $y=0$ so $N$ is faithful. Since $R$ is left $F P F, N$ generates $R$ so $R\left(\left(f_{i}\right)_{i \in I}\right)$ must generate $R g$. It follows that for a fixed $n>0$ there are maps $\sum_{j=1}^{n} R f_{i_{j}} \rightarrow H_{i} \rightarrow 0$ for every $i$. But if $i>n$ we see by Bumbys result $H_{i} \oplus R f_{i} \cong H_{i}$ and $R$ is not Dedekind finite.

Putting the above facts together gives:

THEOREM 9. A regular ring is left FPF iff it is left selfinjective of bounded index.

CoRollary 9.1. A regular ring is left FPF iff it is Morita equivalent to a strongly regular left self-injective ring.

COROLLARY 9.2. A regular ring is left FPF iff it is right FPF.

Proof. By Utumi [13, Thm. 1.4] a strongly regular ring is left self-injective iff it is right self-injective.

ACKNowledgment. I would like to thank the referee for putting the crunch on Proposition 7, to Abraham Zaks for stimulating conversation on the topic and the University of Oregon for its hospitality.

\section{REFERENCES}

1. G. Azumaya, Completely faithful modules and self injective rings, Nagoya Math. J., 27 (1966), 397-708.

2. R. T. Bumby, Modules which are isomorphic to submodules of each other, Arch. der Math., 16 (1965), 184-185.

3. C. Faith, Injective cogenerator rings, Proc. Amer. Math. Soc., 60 (1976), 25-30.

4. - Injective cogenerator rings and a theorem of Tachikawa II, Proc. Amer. Math. Soc., 62 (1977), 15-19.

5. K. R. Goodearl andA. K. Boyle, Dimension theory for nonsingular injective modules, Memoirs Amer. Math. Soc., \#177.

6. I. Kaplansky, Rings of Operators, New York (1968), W. A. Benjamin.

7. B. L. Osofsky, A generalization of quasi-Frobenius rings, J. Algebra, 4 (1966), 373-387.

8. H. Tominaga, Some remarks on $\pi$-regular rings of bounded index, Math. J. Okayama Univ., 4 (1955), 135-144.

9. Y. Utumi, On quotient rings, Osaka Math. J., 8 (1956), 1-18.

10. - A note on an inequality of Levitzki, Proc. Japan Acad., 33 (1957), $249-251$.

11. - On continuous rings and self-injective rings, Trans. Amer. Math. Soc.,

118 (1965), 158-173.

12. - Self injective rings, J. Algebra, 6 (1967), 56-64. 
13. Y. Utsumi, On rings of which any one sided quotient rings are two sided, Proc. Amer. Math. Soc., 14 (1963), 141-147.

Received September 16, 1977 and in revised form March 6, 1975.

UNIVERSITY OF BRITISH COLUMBIA

Vancouver, B. C. Canada V6T 1W5 


\section{PACIFIC JOURNAL OF MATHEMATICS}

EDITORS

RICHARD ARENS (Managing Editor)

University of California

Los Angeles, California 90024

C. W. Curtis

University of Oregon

Eugene, OR 97403

C. C. MOORE

University of California

Berkeley, CA 94720
J. DUGUNDJI

Department of Mathematics University of Southern Californı Los Angeles, California 90007

R. Finn and J. Milgram Stanford University Stanford, California 94305

\section{ASSOCIATE EDITORS}

E. F. BECKENBACH

B. H. NEUMANN

F. WOLF

K. YoSHIDA

\section{SUPPORTING INSTITUTIONS}

UNIVERSITY OF BRITISH COLUMBIA
CALIFORNIA INSTITUTE OF TECHNOLOGY
UNIVERSITY OF CALIFORNIA
MONTANA STATE UNIVERSITY
UNIVERSITY OF NEVADA, RENO
NEW MEXICO STATE UNIVERSITY
OREGON STATE UNIVERSITY
UNIVERSITY OF OREGON

UNIVERSITY OF SOUTHERNEALIFORNIA STANFORD UNIVERSITY UNIVERSITY OF HAWAII UNIVERSITY OF TOKYO UNIVERSITY OF UTAH WASHINGTON STATE UNIVERSITY UNIVERSITY OF WASHINGTON 


\section{Pacific Journal of Mathematics \\ Vol. 79 , No. 1 \\ May, 1978}

Teófilo Abuabara, A remark on infinitely nuclearly differentiable

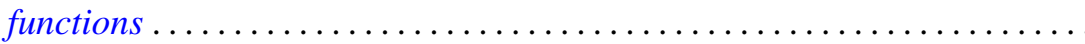

David Fenimore Anderson, Projective modules over subrings of $k[X, Y]$

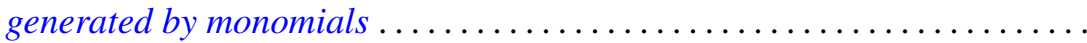

Joseph Barback and Thomas Graham McLaughlin, On the intersection of

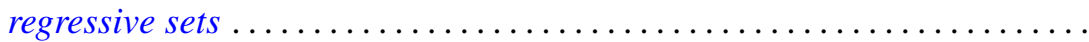

Murray Bell, John Norman Ginsburg and R. Grant Woods, Cardinal inequalities for topological spaces involving the weak Lindelof

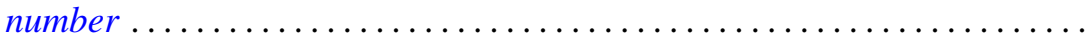

Laurence Richard Boxer, The space of ANRs of a closed surface ............

Zvonko Cerin, Homotopy properties of locally compact spaces at

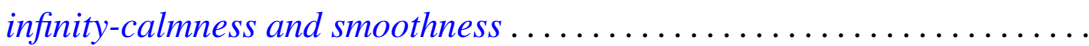

Isidor Fleischer and Ivo G. Rosenberg, The Galois connection between partial functions and relations..................................

John R. Giles, David Allan Gregory and Brailey Sims, Geometrical implications of upper semi-continuity of the duality mapping on a Banach

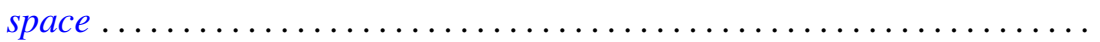

Troy Lee Hicks, Fixed-point theorems in locally convex spaces ............ Hugo Junghenn, Almost periodic functions on semidirect products of transformation semigroups ........................

Victor Kaftal, On the theory of compact operators in von Neumann algebras. II . . . .

Haynes Miller, A spectral sequence for the homology of an infinite

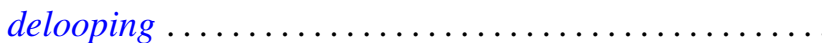

Sanford S. Miller, Petru T. Mocanu and Maxwell O. Reade, Starlike integral operators...

Stanley Stephen Page, Regular FPF rings ...............

Ghan Shyam Pandey, Multipliers for C, 1 summability of Fourier series ...

Shigeo Segawa, Bounded analytic functions on unbounded covering surfaces...

Steven Eugene Shreve, Probability measures and the C-sets of

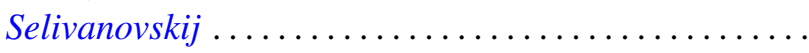

Tor Skjelbred, Combinatorial geometry and actions of compact Lie groups....

Alan Sloan, A note on exponentials of distributions.

Colin Eric Sutherland, Type analysis of the regular representation of a nonunimodular group.

Mark Phillip Thomas, Algebra homomorphisms and the functional

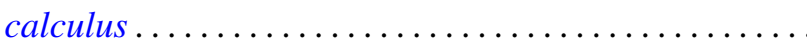

Sergio Eduardo Zarantonello, A representation of $H^{p}$-functions with

$0<p<\infty$. 